\title{
Special issue on Intelligent Fog and Internet of Things (loT)-Based Services
}

\author{
Farookh Khadeer Hussain ${ }^{1} \cdot$ Wenny Rahayu ${ }^{2} \cdot$ Makoto Takizawa $^{3}$
}

Published online: 14 May 2021

(c) Crown 2021

Fog networks are becoming increasingly mainstream in the past few years. They are being used to deploy and deliver services at scale. The need to deliver scalable performance and reliability on networks has been amplified even further by the advancement and popularity of Internet of Things services. However, several challenges need to be addressed, such as but not limited to interfacing the Fog networks with other traditional networks in a seamless manner. Further outstanding research challenges in this area that require attention include approaches to providing QoS guarantees in delivering services, trustworthy service selection, fog orchestration etc. These research challenges would increasingly leverage Artificial Intelligence (AI) based algorithms and mechanisms to address these challenges.

The purpose of this special issue was to stimulate research in this space. In response to the call for papers, we received many submissions. After the review process, five papers were accepted for publication in the special issue.

In the first paper by Cuka et al. [3], the authors proposed an intelligent fuzzy logic-based approach for node elimination followed by node selection in Opportunist Networks (OppNets). OppsNets can be one of the topologies used to deploy and provision Internet of Things (IoT)-based services. The significance of node elimination and node selection in OppNets is amplified by the lack of a path between the source node and the target node. Hence, in OppNets, the path is created in an ad-hoc manner 'on-the-fly' manner. The inherent challenge is to eliminate nodes from amongst the available pool of nodes and select one node to transfer or convey the message to the target node. To achieve the dual tasks of node elimination and node selection, the authors propose using a fuzzy logic-based approach. The efficiency of the proposed fuzzy logic-based approach is demonstrated using simulation experiments.

Farookh Khadeer Hussain

Farookh.Hussain@uts.edu.au

Wenny Rahayu

W.Rahayu@latrobe.edu.au

Makoto Takizawa

makoto.takizawa@computer.org

1 School of Computer Science, University of Technology Sydney, 15 Broadway, Ultimo, NSW, Australia

2 Engineering and Mathematical Sciences, La Trobe University, Victoria 3086, Australia

3 Faculty of Science and Engineering, Hosei University, 3-7-2 Kajino-Cho, Koganei-Shi, Tokyo 184-8584, Japan 
In the second paper, Chen et al. [1] propose an intelligent approach for carrying out Internet of Things (IoT)-based firmware performance analysis to enhance the security of the IoT devices. Dynamic instrumentation is a commonly used technique in software testing. However, typically dynamic instrumentation approaches are resource-intensive and have had little application or use in IoT devices. In this paper, Chen et al. propose an analysis tool (IoTDIT) for runtime error detection in IoT-based services to address this issue.

In the third paper, Adhinugraha et al. [2] propose using a Mesh network as a topology for delivering IoT services. Unlike other topologies, gateways play a critical role in interfacing with the Cloud in the Mesh topology. To ensure network connectivity and availability during failover, the router must always have backup gateways in the Mesh topology to maintain mesh robustness. The authors propose using an Order-k Voronoi diagram to identify and maintain the k-nearest facilities to ensure reliability and fault tolerance of the overall IoT service. Experimental results demonstrate the accuracy and robustness of the proposed Order-k Voronoi approach.

In the fourth paper, AlHadhrami et al. [5] carried a thorough and systematic review of the extant literature in Distributed Denial of Service (DDoS) attacks in IoT-based services. The primary objective of systematically reviewing the extant literature in this space was to identify pressing and outstanding challenges in DDoS that need research attention. DDoS attacks are one of the significant types of security attacks carried out on IoT-based services. DDoS attacks utilize the limited resources of IoT devices, such as storage limitation and network capacity, thereby constraining the delivery of the IoT-based application.

In the final paper by Kim et al. [4], the authors proposed an intelligent method for carrying out image processing and analysis on the social media platforms used to share images from embedded camera on mobile phones. The salient feature of the proposed method is that based on the uploaded images, the proposed method can intelligently provide recommendations by a given target user. The proposed method is based on the Latent Dirichlet Allocation (LDA) for carrying out the target user's analysis. The authors have carried out extensive testing and experimentation that demonstrate the superiority of the proposed method over the other state-of-the-art methods. This intelligent method in image processing will contribute to the advancement of image analytics from IoT or mobile devices.

In concluding our editorial, we would like to thank Professor Yanchun Zhang for providing us with the opportunity to guest-editing this special issue. This special issue would not have been possible without his strong encouragement and leadership. We would also like to thank the authors of all the submitted papers for submitting their papers for consideration in this special issue. Finally, we would like to acknowledge our gratitude to the reviewers for their efforts in reviewing the papers submitted to this SI.

\section{References}

1. Adhinugraha, K., Rahayu, W., Hara, T. et al. Backup gateways for IoT mesh network using order-k hops Voronoi diagram.

2. Al-Hadhrami, Y., Hussain, F.K. DDoS attacks in IoT networks: a comprehensive systematic literature review.

3. Chen, C., Ma, J., Qi, T. et al. Firmware code instrumentation technology for internet of things-based services.

4. Cuka, M., Elmazi, D., Ikeda, M. et al. Application of fuzzy logic for IoT node elimination and selection in opportunistic networks: performance evaluation of two fuzzy-based systems. 
5. Kim, K., Kim, J., Kim, M. et al. User interest-based recommender system for image-sharing social media.

Publisher's Note Springer Nature remains neutral with regard to jurisdictional claims in published maps and institutional affiliations. 